\title{
Symptomatic interdural cerebrospinal fluid collections after surgery for idiopathic spinal cord herniation: illustrative case
}

\author{
Yasufumi Ohtake, MD, Makoto Senoo, MD, Mamoru Fukuda, MD, Yuuki Ishida, MD, Ryunosuke Yoshihara, MD, Kohei Ishikawa, MD, \\ Tomoki Fuchizaki, MD, Tomoaki Ishizuka, MD, Naoyasu Okamura, MD, and Hirohiko Nakamura, MD, PhD
}

Department of Neurosurgery, Spine \& Peripheral Nerve Center, Nakamura Memorial Hospital, Sapporo, Japan

BACKGROUND Idiopathic spinal cord herniation (ISCH) is very rare. Some reports have described postoperative ventral cerebrospinal fluid (CSF) collections in patients with ISCH; however, such collections are asymptomatic in most patients, and there is no consensus regarding whether they are part of the natural history or a complication.

OBSERVATIONS A 30-year-old man with ISCH underwent direct closure of a duplicated dura mater. Eight months postoperatively, he developed reworsening of right lower limb paresis and new severe right arm pain and paresis. Three-dimensional magnetic resonance imaging revealed ventral CSF collections, which the authors judged as the responsible lesions. The authors initially considered these collections to be present in the epidural space, extradurally compressing the dural sac and resulting in myelopathy. An epidural blood patch failed; however, a CSF drainage test resulted in dramatic improvement. The authors therefore determined that the CSF collections were located in the interdural space, not the epidural space. A lumboperitoneal (LP) shunt was performed to reduce the CSF pressure. The patient's symptoms improved immediately postoperatively. He had developed no recurrence of symptoms 6 months after surgery.

LESSONS Ventral interdural CSF collections after ISCH surgery can cause reworsening of myelopathy and may be cured by a LP shunt to control CSF pressure.

https://thejns.org/doi/abs/10.3171/CASE21232

KEYWORDS cerebrospinal fluid collections; idiopathic spinal cord herniation; lumboperitoneal shunt

Idiopathic spinal cord herniation (ISCH) is very rare, but reports of this condition have recently been increasing with the development of magnetic resonance imaging (MRI). ${ }^{1} \mathrm{ISCH}$ is caused by herniation of the spinal cord through an anterior dural defect. The surgical indication for ISCH is the presence of progressive and/or severe neurological deficits. $^{2}$ Although several surgical procedures for ISCH have been reported, such as direct closure of the dural defect, ${ }^{3}$ placement of a dural graft, ${ }^{4}$ and enlargement of the dural defect, ${ }^{5}$ there is no consensus regarding which procedure is most suitable. Furthermore, few studies have investigated the long-term outcomes and MRI findings of postoperative changes in the spinal cord or surrounding structures. ${ }^{1}$

In recent years, several reports have described postoperative ventral cerebrospinal fluid (CSF) collections in patients with $\mathrm{ISCH}^{1,6,7}$ However, these collections are asymptomatic in most patients, and whether they are part of the natural history or a complication remains unclear.

Herein, we report a case of symptomatic ventral CSF collections after surgery for ISCH. The patient presented with severe pain and recurrence of myelopathy. He dramatically recovered after performance of a lumboperitoneal (LP) shunt.

\section{Illustrative Case}

A 30-year-old man presented with a 1-year history of right lower limb weakness, sensory disturbance, and high back pain. As the symptoms progressed, he visited the orthopedics department at another institute and was referred to a neurologist at our institute for a thorough examination. Clinical examination revealed mild right

ABBREVIATIONS CSF = cerebrospinal fluid; EDBP = epidural blood patch; ISCH = idiopathic spinal cord herniation; $L P=$ lumboperitoneal; MASA = multisegmental amyotrophy; MRI = magnetic resonance imaging.

INCLUDE WHEN CITING Published October 4, 2021; DOI: 10.3171/CASE21232.

SUBMITTED April 12, 2021. ACCEPTED August 2, 2021.

(C) 2021 The authors, CC BY-NC-ND 4.0 (http://creativecommons.org/licenses/by-nc-nd/4.0/). 


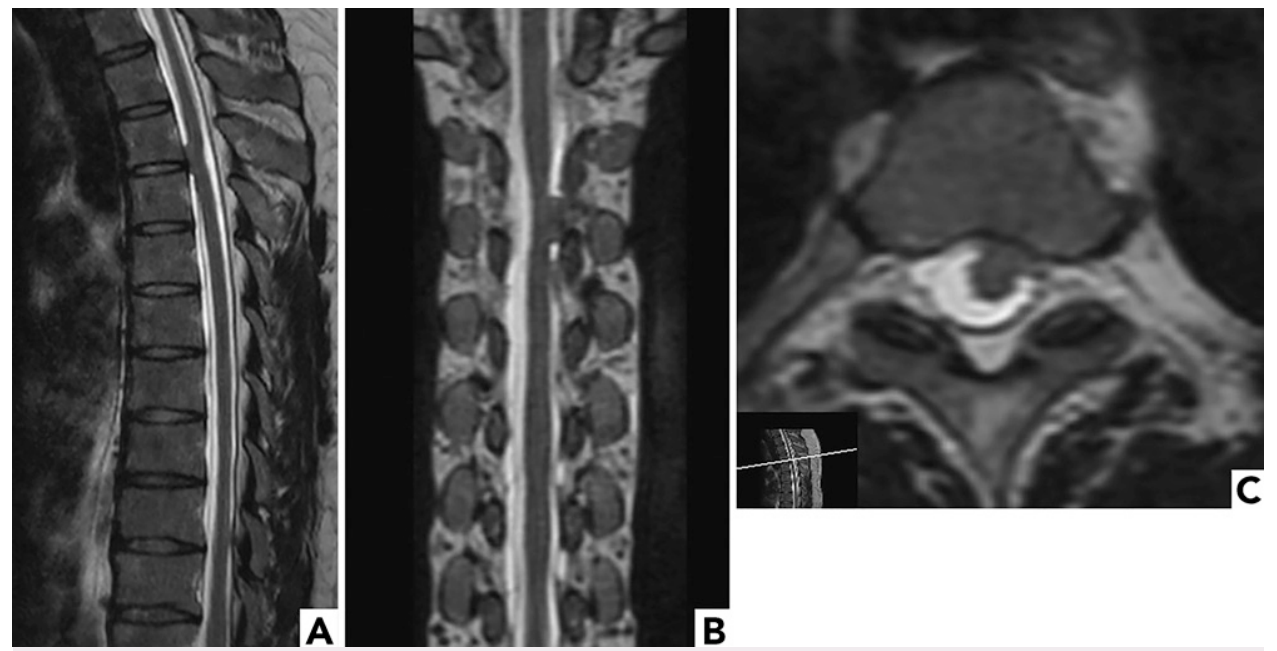

FIG. 1. Preoperative MRI findings of the thoracic spine. A: Three-dimensional T2-SPACE MRI revealed ISCH at T3-4. B: The coronal view confirmed the left and laterally herniated spinal cord. C: The axial image showed the anterolaterally displaced spinal cord.

lower limb weakness, hyperalgesia with the sensory level below T6, a right Babinski sign, and bilateral lower extremity hyperreflexia.

Spinal MRI, performed with a three-dimensional T2-sampling perfection with application-optimized contrast using different flipangle evolution (T2-SPACE ${ }^{8}$ ) sequence, revealed an anteriorly displaced spinal cord at T3-4, and we diagnosed ISCH (Fig. 1).

\section{Surgery}

The patient underwent T3-4 laminectomy and left paramedian durotomy. The spinal cord and arachnoid membrane were herniated through a left ventral and lateral dural defect occupying the hole (Fig. 2A). We reduced the herniation into the normal position. During the operation, we confirmed duplication of the ventral dura mater (Fig. 2B) and primarily closed the dural defect by filling it with DuraGen (Integra LifeSciences). After surgery, the patient recovered from his symptoms and was discharged with no new deficits; only slight sensory disturbance remained.

\section{Postoperative Course}

At 8 months after surgery, the patient presented with reworsening of the right lower limb paresis and new acute severe right arm pain and upper limb paresis. Conventional two-dimensional T2-weighted imaging showed no lesions, such as cervical disc herniation, hematoma, or an abscess. He was treated conservatively but was then hospitalized because his symptoms gradually worsened. Three-dimensional T2SPACE MRI revealed ventral CSF collections at the C2-T6 level (Fig. 3), and no other pathologies were found that may have caused myelopathy. With reference to a previous report, ${ }^{1,6}$ we considered that these CSF collections were located in the epidural space, extradurally compressing the dural sac and resulting in myelopathy.
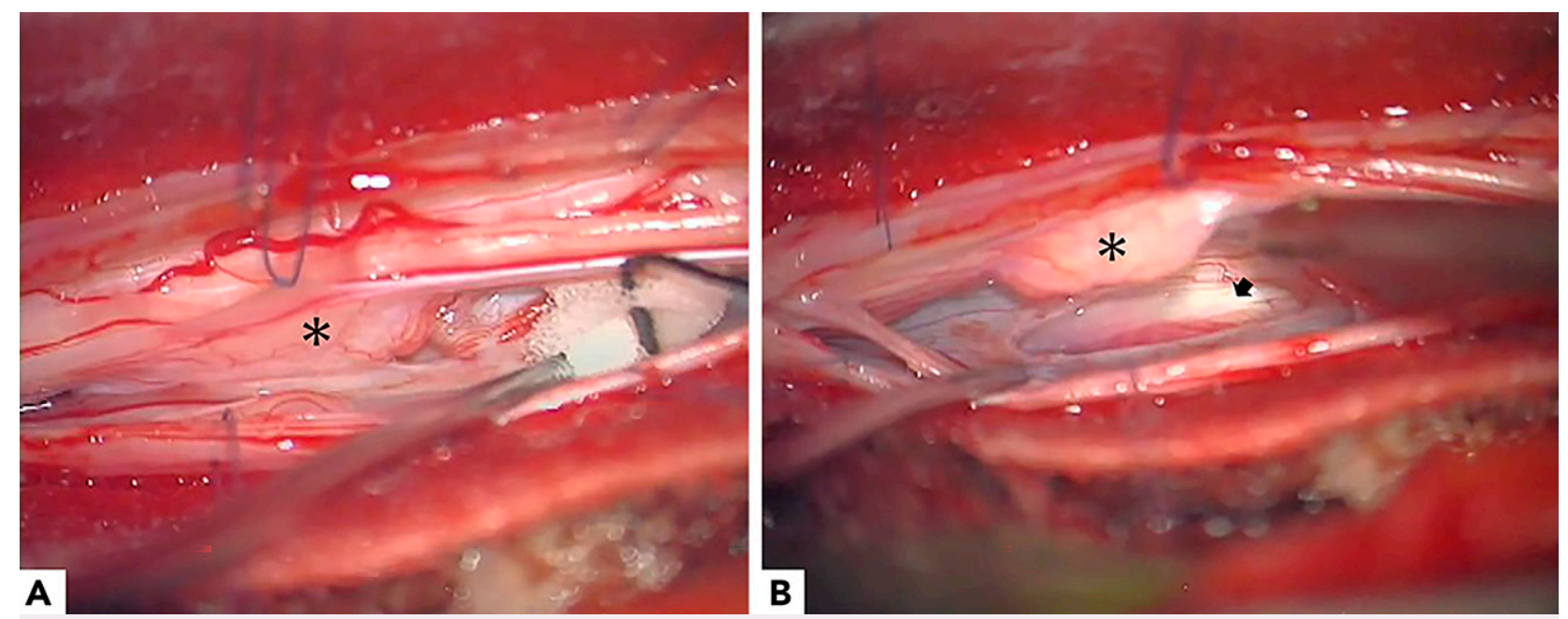

FIG. 2. A: Intraoperative photograph demonstrating evidence of spinal cord herniation (asterisk) through the defect in the anterior dura mater.

B: The arrow indicates the duplication of the dura mater after release of the spinal cord (asterisk) and reduction of cord herniation. 

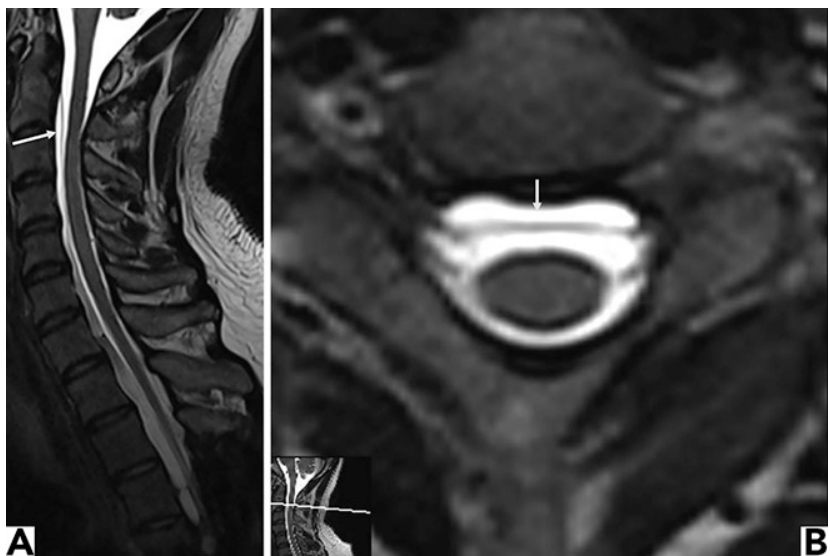

FIG. 3. A: A large ventral CSF collection extending from C2 to T10 was seen on postoperative MRI. B: The white arrows indicate a membrane-like structure that was found to be a part of the dorsal dura mater of the duplicated dura.

Therefore, we performed an epidural blood patch (EDBP) to seal the CSF leakage site.

During the EDBP, the patient complained of severe high back pain, which we considered may have been related to the injection of autologous blood. Only $7 \mathrm{~mL}$ of autologous blood was injected. Postoperative MRI revealed that the injected blood was present in the dorsal space, not the ventral space, despite the fact that the patient had been placed in the prone position. Postoperative MRI showed disappearance of the CSF collection (Fig. $4 A$ and $C$ ), but MRI 9 days after the EDBP (Fig. $4 B$ and $D$ ) showed recurrence of the ventral CSF collection. The patient's symptoms were temporarily improved, but they reworsened with the CSF recollection.

Based on the patient's clinical course, we determined that the CSF collections were located in the "interdural" space, not the epidural space. Temporary improvement was achieved by mechanical compression of the ventral pouch (Fig. 5B).

A CSF drainage test by lumbar puncture resulted in dramatic improvement; therefore, an LP shunt was performed using a programmable valve (CERTAS Plus Programmable Valve; Integra LifeSciences) to reduce the CSF pressure.

Immediately after the operation, the patient's symptoms improved, and he was discharged from the hospital without any assistance. He had developed no recurrence of symptoms 6 months after surgery.

\section{Discussion}

$\mathrm{ISCH}$ is very rare, but it is being increasingly reported with the development of MRI. ${ }^{3}$ Nevertheless, very few reports have described the postoperative course and MRI findings of ISCH.

$\mathrm{ISCH}$ is caused by herniation of the spinal cord through an anterior dural defect. Most surgeons choose surgical treatment for ISCH with progressive and/or severe neurological deficits. ${ }^{2}$ However, although several surgical procedures for ISCH have been described (e.g., direct closure of the dural defect, ${ }^{3}$ placement of a dural graft, ${ }^{4}$ and enlargement of the dural defect ${ }^{5}$ ), no consensus has been reached regarding which procedure is most suitable.

In recent years, several reports have described postoperative ventral CSF collections, ${ }^{1-3,6}$ indicating that they may be caused by subsequent CSF leakage through the dural defect. The first report of postoperative ventral CSF collection was written by Nakamura et al. ${ }^{6}$ and described sixteen patients with $\mathrm{ISCH}$ treated by
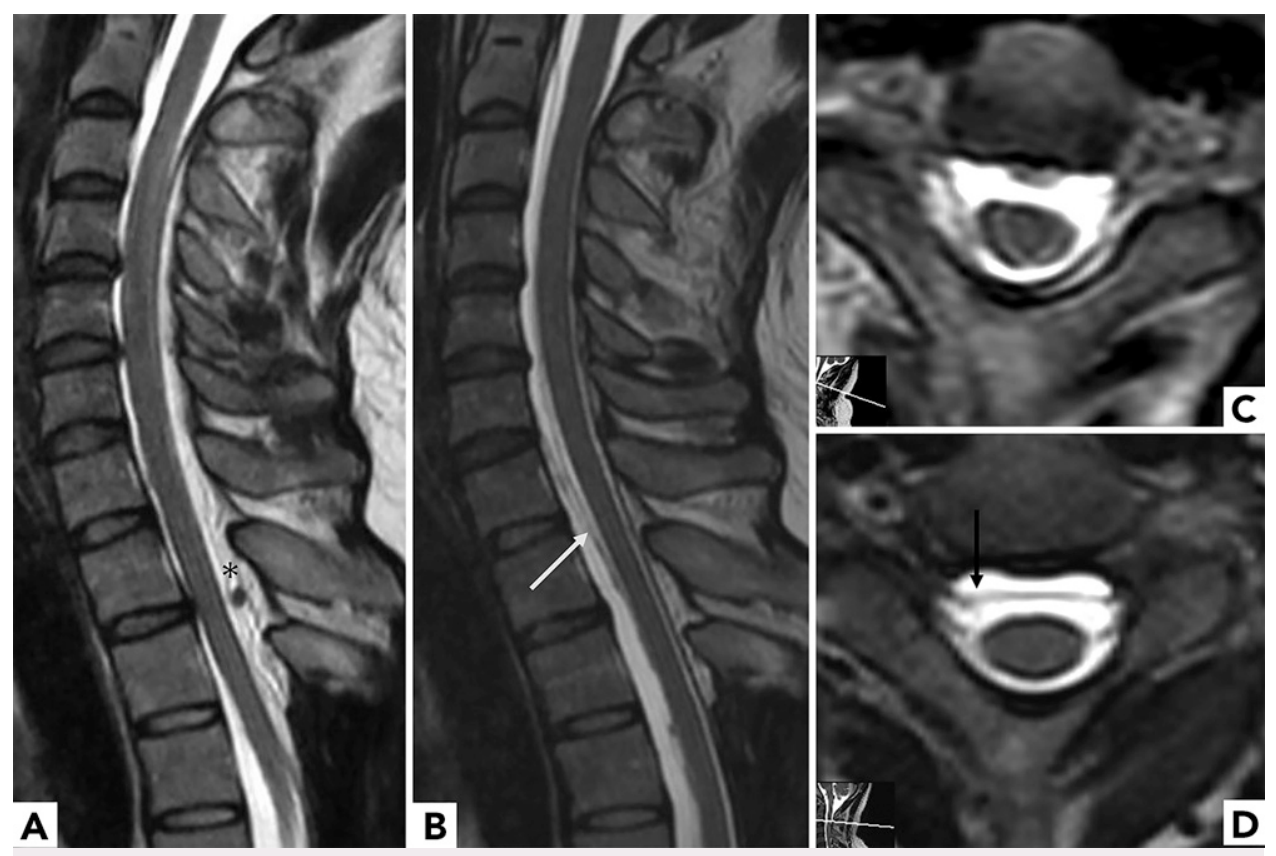

FIG. 4. MRI findings of the cervical spine after EDBP. A: The asterisk indicates the injected autologous blood, and the CSF collection disappeared. B: MRI 9 days after EDBP showed recurrence of the ventral CSF collection (white arrow). C: The axial image showed disappearance of the dura mater after EDBP. D: MRI 9 days after EDBP showed recurrence of the ventral membrane (black arrow). 
A

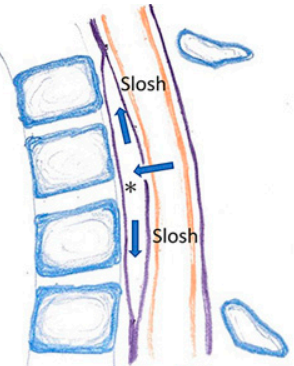

B
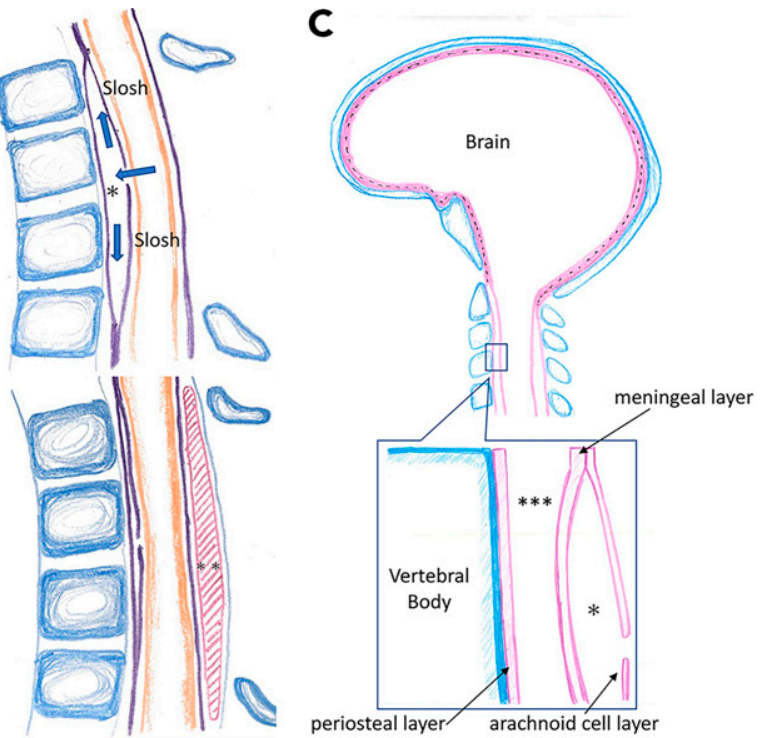

FIG. 5. Schema of dural pouch filling with CSF collection. The single asterisk indicates the ventral pouch filled with CSF. The double asterisk indicates the autologous blood. A: Image after the ISCH operation. The herniated spinal cord was reduced, and a slight hole remained. CSF flowed in through this hole and tore the duplicated dura mater. B: After EDBP, the dorsally placed autologous blood mechanically compressed the ventral pouch, preventing CSF from flowing in. As injected autologous blood was melted and vanished, CSF flowed into the pouch again, and the patient's myelopathy reworsened. C: Schema of cranial and spinal dura mater. The triple asterisk indicates the epidural space in the spinal canal between the periosteal layer and the meningeal layer. The single asterisk indicates the interdural space composed of duplicated dura mater.

enlargement of the dural defect. However, there are no symptoms associated with ventral CSF collection. Some authors have reported that simple dural enlargement may lead to postoperative anterior CSF collection and cause complications; thus, they recommended the use of an anterior dural patch. ${ }^{1,2}$

Gaudino et al. ${ }^{1}$ reported the postoperative MRI findings after treatment of ISCH in twelve patients with a follow-up period of 7.8 years (range, 2-23 years). The authors found ventral CSF collections in all patients in the early phase after surgery, and almost all collections disappeared gradually over time. However, no patients underwent additional treatment, and three patients who were treated with enlargement of the dural defect showed new neurological symptoms at the late clinical follow-up. The authors stated that these symptoms, including positional headache (worse in an upright position), new low back pain, and sensorineural hearing loss due to pial siderosis, were potentially long-standing complications of $\mathrm{ISCH}$ surgery and had been caused by CSF collections. ${ }^{1}$

To our knowledge, only one report has described additional treatment of postoperative ventral CSF collection. Grobelny et al. ${ }^{7}$ reported that the patient underwent multiple surgeries for ISCH and finally developed positional headache and recurrent paraparesis. MRI revealed a ventral CSF collection causing retropulsion of the spinal cord at the operative site and at a remote level. The authors performed spinal fusion surgery to correct the focal kyphosis and direct repair of the dural defect with a dural allograft (Durepair; Medtronic). ${ }^{7}$

\section{Observations}

No previous report has described the treatment of postoperative ventral CSF collection by an LP shunt. In the present case, we initially considered that the patient's CSF collections were located within the epidural space, extradurally compressing the dural sac and resulting in myelopathy. Therefore, we performed an EDBP to seal the CSF leakage site; however, this led to only transient relief, and autologous blood failed to reach the ventral canal.

We suspect that the EDBP was ineffective because the CSF collections were present in the "interdural" space, and the CSF flow through the checked valve hole tear in the inter-dura mater caused severe pain and recurrence of myelopathy.

In the cranial cavity, the dura mater is composed of two layers: the periosteal layer and the meningeal layer. Caudal to the foramen magnum, these two layers of collagenous connective tissue separate from each other, and the externally located periosteal layer transitions to the periosteum covering the inside of the spinal canal. Only the internally located meningeal layer wraps around the spinal cord, and the epidural space exists between these two layers ${ }^{9}$ (Fig. 5C).

Reina et al. ${ }^{10}$ described the ultrastructural findings of the spinal dura mater. According to this report, $90 \%$ of the outer layer of the spinal dura mater is a fibrous structure, and $10 \%$ of the internal layer is formed by the arachnoid cell layer. Therefore, in our case, we considered that CSF had collected between these two layers, and we defined this space as the interdural space (Fig. 5).

In addition, many cases of duplicated dura mater have been reported in the intraoperative findings of idiopathic spinal cord herniation, similar to our case. Some of these reports described direct resection and pathologic examination of the duplicated dura mater with histological findings similar to those of the dorsal dura and no evidence of inflammation. ${ }^{2,5}$ We believe that these findings support our hypothesis.

The CSF drainage test indicated that the intraspinal CSF pressure was decreasing and that the CSF leakage through the checked valve hole and interdural pouch was shrinking (Fig. 5B). Therefore, we considered that if the CSF pressure could be controlled, the patient's condition would be cured, and we performed an LP shunt. The advantages of this procedure are that it is reversible and less invasive. Direct repair or placement of a dural patch requires a more delicate operation, and some authors have reported postoperative neurological deterioration. ${ }^{11}$ If the defect is obstructed or the LP shunt is no longer needed, it can be removed even long after surgery.

Some of the symptomatic dural abnormalities in the present case may be classified as "duropathies." In 2012, Kumar ${ }^{12}$ proposed the concept of duropathies for pathologies that present with neurological symptoms due to fine defects or damage to the dura mater in the spinal canal. Duropathies include spontaneous intracranial hypotension, superficial siderosis, $\mathrm{ISCH}$, and multisegmental amyotrophy (MASA). MASA is considered to be caused by longterm compression of an extradural CSF collection and causes degeneration at the anterior horn of the spinal cord. ${ }^{13}$ The mechanism in the present case seemed to be similar to that of MASA.

\section{Lessons}

We have reported a case of symptomatic interdural cerebrospinal fluid collections after surgery for ISCH. We experienced complications that have not been described in previous cases of ISCH 
surgery; fortunately, our patient successfully recovered. An LP shunt was useful to control the CSF pressure.

Our findings have some limitations. Our hypothesis was made by indirect findings that the CSF drainage test was positive, EDBP was ineffective, and the duplicated dura was found in the first operation; therefore, we did not directly observe an interdural defect. Although we confirmed the absence of spontaneous intracranial hypotension or superficial siderosis before surgery, there may have been neurological deterioration.

\section{Acknowledgment}

We thank Angela Morben, DVM, ELS, from Edanz Group, for editing a draft of this manuscript.

\section{References}

1. Gaudino S, Colantonio R, Schiarelli C, et al. Postoperative MR imaging of spontaneous transdural spinal cord herniation: expected findings and complications. AJNR Am J Neuroradiol. 2016;37(3):558-564.

2. Maira G, Denaro L, Doglietto F, Mangiola A, Colosimo C. Idiopathic spinal cord herniation: diagnostic, surgical, and follow-up data obtained in five cases. J Neurosurg Spine. 2006;4(1):10-19.

3. Borges LF, Zervas NT, Lehrich JR. Idiopathic spinal cord herniation: a treatable cause of the Brown-Sequard syndrome-case report. Neurosurgery. 1995;36(5):1028-1033.

4. Slavotinek JP, Sage MR, Brophy BP. An unusual spinal intradural arachnoid cyst. Neuroradiology. 1996;38(2):152-154.

5. Watanabe M, Chiba K, Matsumoto M, Maruiwa H, Fujimura $Y$, Toyama $Y$. Surgical management of idiopathic spinal cord herniation: a review of nine cases treated by the enlargement of the dural defect. J Neurosurg. 2001;95(2 Suppl):169-172.

6. Nakamura M, Fujiyoshi K, Tsuji O, et al. Long-term surgical outcomes of idiopathic spinal cord herniation. J Orthop Sci. 2011;16(4):347-351.

7. Grobelny BT, Smith M, Perin NI. Failure of surgery in idiopathic spinal cord herniation: case report and review of the literature. World Neurosurg. 2020;133:318-323.
8. Okamura N, Ohtake Y, Anzai K, et al. Evaluation of cervical spine lesions by the 3D T2-SPACE compared with the 2D T2WI. Spinal Surg. 2020;34:59-65.

9. Schuenke M, Schulte E, Schumacher U, Ross L, Lamperti E. Thieme Atlas of Anatomy: Head, Neck, and Neuroanatomy. 1st ed. Thieme Medical Publishers; 2007:190-191.

10. Reina MA, Franco CD, Prats-Galino A, et al. Ultrastructural anatomy of the spinal meninges and related structures. In: Hadzic $A$, ed. Hadzic's Textbook of Regional Anesthesia and Acute Pain Management. 2nd ed. McGraw Hill; 2017:101-123.

11. Groen RJ, Middel B, Meilof JF, et al. Operative treatment of anterior thoracic spinal cord herniation: three new cases and an individual patient data meta-analysis of 126 case reports. Operative Neurosurgery. 2009;64(3 Suppl):ons145-ons160.

12. Kumar N. Beyond superficial siderosis: introducing "duropathies". Neurology. 2012;78(24):1992-1999.

13. Yagishita A. Definitions and MR imaging of duropathies. Article in Japanese. Rinsho Shinkeigaku. 2021;61(1):1-5.

\section{Disclosures}

The authors report no conflict of interest concerning the materials or methods used in this study or the findings specified in this paper.

\section{Author Contributions}

Conception and design: Ohtake, Fukuda, Nakamura. Acquisition of data: Ohtake, Fukuda, Fuchizaki. Analysis and interpretation of data: Ohtake, Fukuda, Yoshihara, Ishikawa. Drafting the article: Ohtake, Fukuda, Ishikawa. Critically revising the article: Ohtake, Fukuda, Ishikawa. Reviewed submitted version of manuscript: Ohtake, Fukuda. Approved the final version of the manuscript on behalf of all authors: Ohtake. Statistical analysis: Ohtake, Fukuda. Administrative/technical/ material support: Ohtake, Fukuda, Ishida, Ishizuka, Okamura. Study supervision: Ohtake, Senoo, Fukuda, Nakamura.

\section{Correspondence}

Yasufumi Ohtake: Nakamura Memorial Hospital, Hokkaido, Japan. yasotk.arcobaleno@gmail.com. 